\title{
artigo
}

Cunha, A.L.L.; Oliveira, S.G.; Bernardo da Silva, M.R.; Chicharo, S.C.R.; Medeiros, C.S.; Christoffel, M.M.; Silva dos Santos, K.C.F.;

Práticas culturais do primeiro banho do recém-nascido na maternidade: um estudo etnográfico

\section{Práticas culturais do primeiro banho do recém-nascido na maternidade: um estudo etnográfico}

\author{
Cultural practices of the newborn's first bath in the maternity ward: an ethnographic study \\ Prácticas culturales del primer baño del recién nacido en la sala de maternidad: un estudio etnográfico
}

\begin{abstract}
RESUMO
O presente estudo tem como objetivo descrever as práticas culturais do primeiro banho do recém-nascido realizado pela equipe de enfermagem no alojamento conjunto e discutir como essas práticas influenciam o cuidado materno. Trata-se de uma pesquisa qualitativa, com abordagem etnográfica, com 21 profissionais de enfermagem. Os dados foram coletados mediante 0 uso de diário de campo, observação participante e entrevistas semiestruturadas. As categorias temáticas depreendidas foram: A dimensão biológica do banho e 0 banho institucionalizado na maternidade. Os resultados mostraram que valorizar a cultura familiar dentro de uma rotina hospitalar aproxima esta da equipe de enfermagem aliando os saberes popular e científico, desconstruindo o paradigma biomédico. A humanização do atendimento supõe encontro entre sujeitos que compartilham saber, poder e experiência vivida, implicando em transformações políticas, administrativas e subjetivas.
\end{abstract}

DESCRITORES: Recém-Nascido; Alojamento Conjunto; Humanização da Assistência.

\section{ABSTRACT}

The present study aims to describe the cultural practices of the first bath of the newborn performed by the nursing staff in the rooming and discuss how these practices influence maternal care. This is a qualitative research, with ethnographic approach, with 21 nursing professionals. Data were collected using field diary, participant observation and semi-structured interviews. The thematic categories were: The biological dimension of the bath and the institutionalized bath in the maternity ward. The results showed that valuing family culture within a hospital routine brings it closer to the nursing team, combining popular and scientific knowledge, deconstructing the biomedical paradigm. The humanization of care presupposes meeting between subjects who share knowledge, power and lived experience, implying political, administrative, and subjective transformations. DESCRIPTORS: Newborn; Joint Accommodation; Humanization of Assistance.

\section{RESUMEN}

El presente estudio tiene como objetivo describir las prácticas culturales del primer baño del recién nacido realizado por el personal de enfermería en la habitación y discutir cómo estas prácticas influyen en la atención materna. Esta es una investigación cualitativa, con enfoque etnográfico, con 21 profesionales de enfermería. Los datos fueron recolectados usando un diario de campo, observación participante y entrevistas semiestructuradas. Las categorías temáticas fueron: La dimensión biológica del baño y el baño institucionalizado en la sala de maternidad. Los resultados mostraron que valorar la cultura familiar dentro de la rutina de un hospital lo acerca al equipo de enfermería, combinando conocimiento popular y científico, deconstruyendo el paradigma biomédico. La humanización de la atención supone una reunión entre sujetos que comparten conocimientos, poder y experiencias vividas, lo que implica transformaciones políticas, administrativas y subjetivas.

DESCRIPTORES: Recién Nacido; Alojamiento Conjunto; Humanización de la Atención.

RECEBIDO EM: 14/01/2020 APROVADO EM:15/01/2020

\section{Adriana Loureiro da Cunha}

Enfermeira. Psicóloga. Mestre em Enfermagem pela EEAN/UFRJ. https://orcid.org/0000-0002-6971-4357

\section{Samara Gonçalves de Oliveira}

Enfermeira. Especialista em saúde da família pelo programa de residência em enfermagem em saúde da família da Secretaria Municipal de Saúde do Rio de Janeiro. https://orcid.org/0000-0002-4532-9489 


\section{Maria Regina Bernardo da Silva}

Enfermeira. Mestre em saúde da família. Docente da Universidade Castelo Branco. https://orcid.org/0000-0002-3620-3091

\section{Sandra Conceição Ribeiro Chicharo}

Enfermeira. Especialista em terapia intensiva. Especialista em docência do ensino superior. Mestre em ensino e saúde pelo programa acadêmico de ciências do cuidado e saúde. Docente da UniSãoJosé e da Universidade Castelo Branco. https://orcid. org/0000-0002-1487-0088

\section{Claudia da Silva de Medeiros}

Enfermeira. Mestre em saúde da família. Universidade Castelo Branco. https://orcid.org/0000-0003-0471-0731

\section{Marialda Moreira Christoffel}

Enfermeira. Professora Adjunta da Escola de Enfermagem Anna Nery/ Universidade Federal do Rio de Janeiro. Pós-Doutora em Enfermagem. https://orcid.org/0000-0002-4037-8759

\section{Kelly Cristina Freitas da Silva dos Santos}

Mestre em enfermagem e tecnologia. Docente da Universidade Castelo Branco. https://orcid.org/0000-0003-3070-4124

\section{INTRODUÇÃO}

$\mathbf{0}$ período neonatal é um momento de grande vulnerabilidade da vida. Nele, concentram-se riscos biológicos, ambientais, socioeconômicos, culturais e requer cuidados especiais. Desta forma, torna-se relevante, identificar as necessidades em saúde das mulheres e dos recém-nascidos (RNs) no puerpério ${ }^{(1)}$. O cuidado direcionado ao RN tem importância para a redução da mortalidade infantil, que é responsável por $70 \%$ das mortes no primeiro ano de vida da criança, especialmente entre 0-6 dias de vida, onde representa $68 \%$ das mortes neonatais precoce no Brasil ${ }^{(1,2)}$. As principais causas de óbitos neonatais são: a prematuridade, as infecções perinatais e fatores maternos, além de um considerado número de casos de morte preveníveis por ação dos serviços de saúde ${ }^{(2)}$.

Os dados acima indicam a necessidade de aprimorar as práticas de atenção à saúde a essas pessoas, para promover melhorias nos indicadores de saúde. As políticas públicas demonstram como uma de suas metas, a organização da saúde perinatal no país, com destaque para o Programa de Humanização ao Pré-Natal e Nascimento (PHPN) e a Rede Cegonha ${ }^{(3)}$, onde apresentam estratégias que propõem mudanças no modelo de atenção ao parto, o uso de protocolos, a vigilância dos indicadores de saúde, associados ao uso de tecnologias simples e efetivas ${ }^{(3,4)}$.

A atenção ao parto no Brasil acontece em $98 \%$ nos hospitais ${ }^{(1)}$. Logo após o nascimento, são realizados inúmeros procedimentos para favorecer e manter a adaptação fisiológica do RN à vida extrauterina. Um processo biológico complexo que envolve modificações funcionais em todos os órgãos e sistemas do neonato, permitindo-lhe viver separado da unidade uteroplacentária. Um dos fatores mais importantes nesta fase de adaptação extrauterina é a manutenção da temperatura corporal ${ }^{(1,5,6)}$.

A fim de que o $\mathrm{RN}$ a se adapte ao novo ambiente, as maternidades devem ter em suas dependências o sistema de alojamento conjunto (AC), onde o recém-nascido irá permanecer durante a sua internação até a alta hospitalar. O AC, traduzido do Inglês "Rooming-in", garantido pela Lei n. ${ }^{\circ} 2068$ de 21 de outubro de 2016, é uma forma de organização institucional onde o recém-nascido sadio permanece ao lado de sua mãe em tempo integral ${ }^{(7)}$. Para ser admitido no $\mathrm{AC}$, o recém-nascido deve estar clinicamente estável: com boa adaptação cardiovascular e respiratória, com capacidade de sucção e controle térmico. Os cuidados realizados exclusivamente pela equipe de enfermagem no alojamento conjunto são: a troca de fraldas, a higiene do coto umbilical e o banho do recém-nascido ${ }^{(5)}$.
$\mathrm{O}$ ato de banhar o recém-nascido imerso numa banheira é muito recente nas maternidades brasileiras. Historicamente, os nascimentos até meados do séc. XIX aconteciam nos domicílios, e os cuidados transcorriam em família, estabelecidos por fortes vínculos humanos e sociais ${ }^{(8)}$. Os primeiros cuidados com o recém-nascido eram ancilares, sendo o corpo do RN banhado em liquídos, como o vinho ou cachaça, limpo com manteiga e outras substâncias oleaginosas e firmemente enfaixado ${ }^{(9)}$. Pouco a pouco, as recomendações médicas foram repassadas às puérperas "ensinando" às mães a substituir as pegajosas abluções com óleo, por "água e sabão". Pode-se perceber que os médicos davam grande ênfase ao asseio corporal. Culturalmente, as mães cuidavam para preservar a função simbólica da sujeira do corpo infantil como uma forma de proteção contra bruxarias $^{(9)}$.

Com o advento dos hospitais-maternidades, no séc. XX, houve uma institucionalização do parto e, assim, os cuidados com o recém-nascido passaram a seguir rotinas hospitalares (uso de nitrato de prata $1 \%$ nos olhos (credé), administração de vitamina $\mathrm{K}$ intramuscular, medidas antropométricas). Havia uma preocupação médica em diminuir os riscos para infecção e também a morbi-mortalidade infantil, o modelo de berçário foi instituído mantendo os recém-nascidos isolados de suas mães e fa- 


\section{artigo}

Cunha, A.L.L.; Oliveira, S.G.; Bernardo da Silva, M.R.; Chicharo, S.C.R.; Medeiros, C.S.; Christoffel, M.M.; Silva dos Santos, K.C.F.

Práticas culturais do primeiro banho do recém-nascido na maternidade: um estudo etnográfico

miliares ${ }^{(5,10)}$. O banho do recém-nascido era contra-indicado até a queda do coto umbilical, acreditando ser esta uma das causas de infecçôes ${ }^{(8,9)}$.

No início da década de 80 , surge a "Síndrome da Imunodeficiência Adquirida” (SIDA), e a descoberta de que uma das formas de transmissão é a vertical, ou seja, de mãe para filho, no momento do parto. Sendo assim, as recomendações para os cuidados com o RN passam a sofrer transformações. Uma delas aponta para o cuidado de lavar o RN imediatamente após o parto, com água corrente e sabão, para evitar o contato prolongado com o sangue e secreções maternas ${ }^{(11)}$.

Recomendações recentes, como o guia prático da Organização Mundial de Saúde (OMS) sobre cuidados pós-natal (2013), sugerem que o banho do recém-nascido deve ser postergado até $24 \mathrm{~h}$ ou mais depois do parto. Se não for possível, por questões culturais, que seja feito após 6 horas de nascido ${ }^{(12)}$. Já a Sociedade Brasileira de Pediatria, através do consenso de cuidado com a pele do RN (2015), recomenda que o banho pode ser diário, com sabão neutro, e que pode ter um intervalo de até 3 dias ${ }^{(13)}$.

Em um estudo internacional Europeu, identifica-se como recomendação quanto a frequência do banho de 1 a 2 vezes por semana, porém ressaltam a realização do mesmo conforme as necessidades e os fatores culturais dos pais ${ }^{(14)}$. Em um outro estudo etnográfico, realizado por pesquisadores norte americanos com 27 mulheres e 16 cuidadoras, de uma área rural do Cambodja em 2015, as questões culturais relativas ao banho do RN foram valorizadas. Os participantes da pesquisa relataram que realizam o banho do RN em uma banheira ao ar livre, numa frequência de 2 vezes por dia, por conta do clima quente local, e geralmente quem realiza este banho são as avós ou um familiar mais velho que a mãe $e^{(15)}$.

O manual de atenção ao recém-nascido $^{(16)}$ recomenda em um de seus protocolos que os pais devem ajudar no primeiro banho e receber informações sobre o recém-nascido e as habilidades para o cuidado. Cita ainda que, o banho melhora a aparência do $\mathrm{RN}$ e é um importante meio para o controle de infecção. Apresentamos a seguinte questão norteadora: Como acontecem as práticas culturais do primeiro banho do recém-nascido realizado pela equipe de enfermagem na maternidade?

Neste contexto, o estudo tem como objetivos: identificar as práticas culturais do primeiro banho do recém-nascido realizado pela equipe de enfermagem na maternidade e discutir como essas práticas influenciam o cuidado materno.

\section{METODOLOGIA}

Trata-se de um estudo de natureza qualitativa ${ }^{(17)}$, cujo projeto foi aprovado pelo Comitê de Ética em Pesquisa da Escola de Enfermagem Anna Nery, Hospital Escola São Francisco de Assis, da Universidade Federal do Rio de Janeiro, sob o parecer n. ${ }^{\circ}$ 089/15. O cenário do estudo foi o alojamento conjunto de um hospital geral localizado na cidade de Duque de Caxias, Rio de Janeiro.

A instituição é referência para atendimento de gestantes de alto risco. $\mathrm{O}$ alojamento conjunto, situado no $2^{\circ}$ andar da unidade, possui 39 leitos para puérperas e seus recém-nascidos. O complexo materno-infantil conta ainda com uma unidade intensiva neonatal, um centro obstétrico e atendimento ambulatorial de puericultura. Em média são realizados cerca de 500 partos por mês.

Os participantes do estudo foram 21 membros da equipe de enfermagem do hospital. Destes, 12 eram técnicos de enfermagem e 9 enfermeiros, escolhidos aleatoriamente e que aceitaram participar do estudo assinando o Termo de Consentimento Livre e Esclarecido (TCLE), em acordo com os preceitos éticos exigidos pela Resolução n. ${ }^{\circ}$ 466/2012 do Conselho Nacional de Saúde (CNS).

O referencial teórico se deu à luz da abordagem etnográfica ${ }^{(18)}$, método clássico que está pautado no olhar, ouvir e escrever $^{(19)}$, sendo essas as três etapas da apreensão dos fenômenos sociais. Foi escolhida considerando que a equipe de enfermagem constitui um grupo cultural que compartilha conhecimento, valores, símbolos e significados. Nesse sentido, foi possível apreender os significados atribuídos pela equipe ao primeiro banho do RN na maternidade e de que forma tais significados influenciam o cuidado materno.

A coleta dos dados aconteceu entre os meses de abril e junho de 2016. A fim de garantir o anonimato e o sigilo dos sujeitos, utilizou-se a identificação da letra $\mathrm{E}$ (Entrevista) seguidas de número indicativo da ordem de realização da entrevista: (E1, E2, E3...). As entrevistas foram realizadas individualmente, o diário de campo foi escrito após cada observação participante que a pesquisadora realizou na sala de banho.

Os dados foram produzidos pela observação participante (olhar), pelas entrevistas com perguntas semiestruturadas gravadas em um aparelho de celular da pesquisadora (ouvir) e pelo diário de campo (escrever) onde foram registradas as impressões da pesquisadora sobre o ambiente, as ações e os comportamentos dos atores sociais envolvidos. As entrevistas foram transcritas na íntegra e, posteriormente, submetidas à análise temática.

A análise dos dados se deu conforme proposto por Minayo ${ }^{(20)}$, que segue os seguintes passos: ordenação, classificação e análise final dos dados. O encerramento do trabalho de campo se deu após atingir a saturação teórica dos dados, uma vez que a coleta é interrompida quando se constata elementos novos para subsidiar a pesquisa não são mais depreendidos a partir do campo de observação, ou seja, quando a interação entre o campo de pesquisa e o investigador não mais oferece elementos para aprofundar a teorização ${ }^{(17)}$.

\section{RESULTADOS}

O material empírico gerado sofreu os 
processos analíticos preconizados pela análise temática ${ }^{(17)} \mathrm{e}$, então, foram construídos os seguintes tópicos de análise: A dimensão biológica do primeiro banho e o Banho institucionalizado no alojamento conjunto.

\section{A dimensão biológica do primeiro banho no alojamento conjunto}

Esta categoria aborda questões relativas à dimensão biológica observadas pelas depoentes antes de realizarem o primeiro banho no RN: a avaliação física, a prevenção da hipotermia, a alimentação do RN, e os cuidados realizados na pele do $\mathrm{RN}$, e relacionada aos fatores comportamentais como: a observação da atividade, reatividade e sonolência. Desta forma, é a equipe que decide se o bebê tem condições para realizar o primeiro banho:

"Eu sempre avalio o RN: se está ativo, se está reativo" (E1)

"Se o bebê estiver hipoativo ou sonolento, o primeiro banho vai ficar para depois" (E 2)

"Se a criança estiver com cianose em extremidades ou batimentos de asa de nariz, são sinais de que não está ainda apta para o primeiro banho" (E3)

Os participantes também apontam a verificação dos sinais vitais como um fator decisivo para realizar o primeiro banho do neonato:

"Se a frequência respiratória do bebê estiver abaixo de $40 \mathrm{irpm}$, eu não arrisco de levar o RN para o primeiro banho" (E3)

"Caso o Rn esteja mantendo a temperatura baixa, menor que $36^{\circ} \mathrm{C}$, eu não faço o banho" (E6)

"Hipotermia pode levar o bebê ao óbito, portanto oriento a equipe para serem atentas $e$ banho somente depois do $R N$ atingir $36,5^{\circ}$ $C$ " $(E 8)$

Os depoentes ressaltaram que é im- portante realizar o primeiro banho se o neonato estiver sendo amamentado, relacionando o controle glicêmico como fundamental para a estabilidade do RN: "Eu vejo, se o bebê está conseguindo mamar, ai sim levo para fazer o primeiro banho" (E3)

"A bipoglicemia pode deixar o bebê fraco e sua temperatura diminuir, portanto não é prudente realizar o primeiro banho do $R n$, nesta condição" (E20)

Alguns profissionais destacaram que se faz necessário o primeiro banho para a limpeza da pele do $\mathrm{RN}$ a fim de retirar as impurezas do parto:

"Tem que limpar tudo, retirar o vérnix: embaixo do pescoço, entre as virilhas, embaixo das axilas. Limpo tudo, é mais higiênico, evita risco de pegarem infecção aqui no hospital" (E8)

"Eu uso o sabão do hospital, ele é neutro, sem cheiro e não prejudicam a pele do bebê "(E8)

\section{0 banho institucionalizado na ma- ternidade}

Esta categoria descreve o manejo do primeiro banho do $\mathrm{RN}$ no alojamento conjunto. Foram abordados os seguintes tópicos: o local do banho, o horário do banho, a relação horas de nascido e a técnica utilizada para banhar o RN. As depoentes relataram que os banhos dos recém-nascidos são realizados numa sala própria, com banheiras individualizadas, água morna, ambiente climatizado, o que consideram um espaço acolhedor. É na sala de banho que a equipe de enfermagem passa a maior parte do tempo, geralmente no período da manhã, o que coincide com o horário que são feitos os banhos:

"Realizamos e ensinamos o banho na sala de banho, é uma forma de acolher o bebê e sua mãe" (E6) "O espaço da sala é destinado ao banho, cada RN tem sua banheira, a água é morna, muito acolhe- dor para a mãe e seu bebê, antes o banho era feito dentro das enfermarias e nem sempre conseguiamos ter água morna para dar o banho (E12)

Já em relação as horas de nascido e o momento em que a equipe realiza o primeiro banho, percebemos que houve divergência na conduta de cada profissional entrevistado:

"Eu prefiro dar o primeiro banho após as 24h de nascido" (E2)

"Geralmente eu faço o primeiro banho depois de 3 horas que o Rn nasceu" (E1)

"Se ele tiver menos de seis horas de nascido, eu não faço o banho" (E21)

Além disso os profissionais referiram que o tipo de banho institucionalizado é o banho humanizado:

"O banho, que ensinamos às mães, é o banho humanizado. Esse banho a gente auxilia a mãe e sempre dizendo para ela como vai ser feito e os benefícios para o bebê" (E2)

"Aqui na unidade o banho humanizado é obrigatório, isso causa um conflito das mães com a equipe, elas nem sempre aceitam fazer o banho assim" (E9)

\section{DISCUSSÃO}

A avaliação do estado geral do RN, é um procedimento que é recomendado pelo Ministério da Saúde, dadas as peculiaridades fisiológicas e anatômicas do neonato, que o diferencia das demais faixas etárias. Desta forma, é recomendado que se avalie o bebê logo nas primeiras 12 horas de vida. Neste momento, deve ser observada a atividade, a intensidade do choro, a movimentação corporal, o estado de hidratação, além dos sinais vitais ${ }^{(1)}$.

O exame físico, que faz parte do processo de enfermagem, representa um 


\section{artigo}

Cunha, A.L.L.; Oliveira, S.G.; Bernardo da Silva, M.R.; Chicharo, S.C.R.; Medeiros, C.S.; Christoffel, M.M.; Silva dos Santos, K.C.F.;

Práticas culturais do primeiro banho do recém-nascido na maternidade: um estudo etnográfico

instrumento que permite ao enfermeiro realizar o diagnóstico e planejar as ações de enfermagem, além de acompanhar e avaliar a evolução do cliente, a fim de promover conforto, segurança e prevenir complicações ${ }^{(21,22)}$.

Sendo assim, a manutenção da temperatura corpórea é um dos fatores determinantes para o sucesso da adaptação extrauterina. Sabe-se que os recém-nascidos são propensos a queda rápida da temperatura corporal através dos mecanismos de convecção, evaporação, condução e radiação. Nos primeiros 10 a 20 minutos de vida, se não houver intervenção para evitar a perda de calor, a temperatura da criança poderá diminuir de $2^{\circ}$ a $4^{\circ} \mathrm{C}$, o que pode contribuir para o aumento da morbimortalidade neonatal ${ }^{(23)}$.

Portanto, o controle térmico rigoroso é proposto pela Organização Mundial de Saúde (OMS), em seu manual de cuidados no período pós natal ${ }^{(12)}$ recomenda aos profissionais de saúde para manterem o ambiente, as mãos e os instrumentos em temperatura adequada, pois o neonato tem pouca capacidade de tolerar as alterações térmicas ambientais. Recomenda igualmente mantê-lo em contato pele a pele com sua mãe, e o uso de gorro, não devendo ser exposto nem ao frio $\left(<35^{\circ}\right.$.C) nem a temperaturas elevadas - $>37^{\circ} \mathrm{C}^{(1,12)}$.

Em relação à alimentação do neonato, as entrevistadas destacaram que estimulam a prática do aleitamento materno, a fim de evitar a hipoglicemia, para que assim possam realizar o primeiro banho do bebê. Explicam ainda que mantém a conduta para aqueles bebês que não podem ser amamentados devido a doenças maternas, onde buscam oferecer o copinho com complemento, se assim prescrito pelo médico.

A hipoglicemia é um achado comum entre os neonatos nas primeiras seis horas de vida, afetando de 3 a $43 \%$ de todos os neonatos, dentre os sinais e sintomas provocados por este distúrbio está a hipotermia(24). Desta forma, a OMS ${ }^{(12)}$ sugere o aleitamento materno na primei- ra meia hora do pós-parto, caso não haja doenças maternas. A amamentação sob livre demanda é a melhor forma de prevenção da hipoglicemia assintomática e a avaliação e apoio contínuo, ainda são as melhores estratégias para evitá-la ${ }^{(16)}$.

Em relação aos cuidados com a pele do neonato, as depoentes relataram realizar uma higiene na pele do bebê assim que é admitido no AC proveniente do centro obstétrico, a fim de eliminar as secreções do parto. $\mathrm{O}$ vérnix caseoso (VC), é uma substância branca que reveste a pele do neonato ${ }^{(25)}$. Sua composição é feita por água $(80 \%)$, proteínas (10\%) e lipídeos (10\%) além de possuir efeitos protetores contra agentes agressivos externos ${ }^{(26)}$. Por várias décadas, nas maternidades ocidentais, era uma prática rotineira retirar todo o $\mathrm{VC}$ dos recém-nascidos, imediatamente após o parto. Esta prática foi reconsiderada e, atualmente, a recomendação é para que o VC fique na pele, por mais de $24 \mathrm{~h}$ pós-parto, a fim de que se promovam a hidratação e a formação do manto ácido, que confere proteção a pele e da formação da camada córnea, a parte mais externa da pele. A exceção segue apenas para as crianças filhas de mulheres portadoras do HIV, onde deve-se banhar este $\mathrm{RN}$ em água corrente imediatamente após o parto ${ }^{(11,13,25)}$.

As recomendações internacionais da Association of Women's Health Obstetric and Neonatal Nurses (AWHONN) reforçam que deve-se evitar banhos diários com uso do sabão. Estes devem ser realizados alternados somente com água e banhos com sabão de $\mathrm{PH}$ neutro e água. O uso de produtos tópicos, tal como o sabão, desfaz o manto ácido, responsável por evitar a colonização bacteriana e promover a retenção de umidade na barreira da pele, promovendo assim a colonização da pele com microorganismos do ambiente hospitalar, favorecendo as infecções hospitalares. O vérnix caseoso não deve ser retirado totalmente no banho, deve-se deixar desprender do corpo do RN naturalmente ${ }^{(26)}$.

Corrobora com este fato, o consen- so da Sociedade Brasileira de Pediatria (SBP) sobre os cuidados com a pele do recém-nascido e alerta que o uso de produtos de limpeza na pele do bebê são tenso-ativos que permitem emulsionar as substâncias da superfície cutânea, eliminando-as. Os agentes de limpeza irritantes são classificados como tenso-ativos alcalinos, já os suaves são os sabonetes com adição de hidratantes e com PH ácido e que podem ser usados sob a forma de barra ou líquidos, destinados às crianças e devem ser enxaguados após o uso ${ }^{(13)}$.

Estudos recentes descreveram que a pele continua a se desenvolver até 12 meses após o nascimento. A pele do neonato é submetida a um processo de adaptação ao ambiente extrauterino, sendo necessários cuidados especiais. $\mathrm{O}$ conhecimento das modificações fisiológicas que ocorrem na pele do RN é fundamental para os profissionais de enfermagem, contribuindo para um cuidado individualizado e seguro, com vistas a contribuir para a manutenção da integridade da pele e melhoria da assistência em saúde a estes clientes ${ }^{(27)}$.

Podemos perceber que o primeiro banho do recém-nascido traz controvérsias entre os profissionais de saúde do cenário de estudo. Permanecem discussões sobre o momento mais apropriado para realizá-lo após o nascimento e como realizar o procedimento estão presentes nas unidades neonatais. As diretrizes sobre cuidados pós-natais da $\mathrm{OMS}^{(12)}$, a Agência de Pesquisa e Qualidade em Saúde $-\mathrm{AHRQ}^{(28)}$ e a Associação de Enfermeiras em Saúde da Mulher, Obstétricas e Neonatais (AWHONN) indicam que o banho inicial do recém-nascido deve ser adiado por até 24 horas após o nascimento, relacionando à estabilização das funções cardiorrespiratórias. A AWHONN fundamenta esta conduta, relacionando à estabilidade da termorregulação do recém-nascido.

Estudos ${ }^{(29,30)}$ reforçam que um atraso no banho pode aumentar o tempo do aleitamento materno exclusivo, diminuindo a separação da mãe e do recém- 
-nascido. Ressaltam que, quando o primeiro banho é precoce, geram-se riscos de danos causados pela hipotermia, prejuízos na amamentação e agravos provocados pela hipoglicemia.

A cultura institucional legitima o ritmo padronizado da unidade do estudo. Porém, em alguns depoimentos, observou-se que o primeiro banho realizado no RN, a equipe de enfermagem leva em consideração o que é preconizado pela $\mathrm{OMS}^{(12)}$, no tocante à realização do primeiro banho após $6 \mathrm{~h}$ de vida da criança. A exceção ficará para os casos em que o RN está banhado em mecônio ou é filho de mulher portadora do vírus da hepatite $B$, herpes vírus ou HIV, com o objetivo de remover resíduos de sangue materno, minimizando a exposição do Rn e da equipe a esses agentes patológicos ${ }^{(31,32)}$.

Outro aspecto apontado pelas participantes foi a técnica do banho humanizado, escolhida para realizar e ensinar às puérperas. A técnica do banho relatada pela equipe depoente, o banho humanizado, é uma técnica adaptada da cultura Japonesa, cujo o objetivo é manter o RN calmo e seguro, mimetizando o ambiente intra-uterino. Inicialmente, deve-se em manter o ambiente aquecido e preparar os materiais. Após o preparo, o RN deve ser despido, enrolado em uma fralda ou cueiro e colocado na água. Durante a execução do banho, deve-se descobrir primeiro um lado, lavar e em seguida cobri-lo novamente, repetindo o mesmo procedimento no lado oposto, para que o bebê se habitue à água. O RN será completamente despido apenas no momento de lavar a parte posterior ${ }^{(33,34)}$.

Posteriormente, o bebê deve ser retirado da água enrolado em um cueiro seco, permanecendo próximo de quem executa a técnica. Posteriormente, o RN é vestido. É importante ressaltar que durante a técnica, o diálogo entre quem executa o banho e o RN é fundamental, sugerindo-se inclusive o uso de cantigas $^{(33,34)}$. No contexto do estudo, ensina-se à mãe o banho humanizado, pois considera-se que este tipo de banho na criança seja algo prazeroso, e que deva lembrar o ambiente líquido e quente característico do útero materno ${ }^{(34)}$.

De um modo geral, os depoimentos demonstraram a importância de um atendimento qualificado à mulher e ao neonato no puerpério. Entretanto, os profissionais devem reconhecer as diferenças culturais, de valores e as experiências vividas por cada mulher e suas famílias. Desenvolver uma sensibilidade cultural, favorecendo uma aproximação e congruência entre as práticas do cuidado popular e profissional, buscando compartilhar saberes e experiências entre os grupos ${ }^{(33)}$.

\section{CONCLUSÃO}

No presente estudo, evidenciaram-se aspectos onde a cultura biomédica leva a equipe de enfermagem a preocupar-se com os fatores fisiológicos de adaptação extrauterina do RN. É muito comum que se adotem critérios centrados em seus conhecimentos técnico-científicos. Desta forma, a rotina institucional limita a mulher e sua família a um só modelo de cuidado, ancorado na cultura profissional.

$\mathrm{O}$ alojamento conjunto torna-se um cenário ideal para a aprendizagem da puérpera em relação aos cuidados com o RN, dentre eles o banho. Haja visto que muitas apresentam medo e insegurança frente a esse cuidado.

Porém, nem sempre os profissionais reconhecem o saber das mães em relação aos cuidados com os seus filhos, imputando-lhes tarefas para que apenas executem, sem inclui-las como protagonistas, esquecendo que isto é fundamental para a manutenção do vínculo afetivo entre pai, mãe e filho.

$\mathrm{O}$ presente trabalho não almejou findar a discussão sobre esse tema. Para superar as limitações do estudo, é necessário replicar a pesquisa em diferentes cenários da prática de atenção à saúde da criança e de seus familiares, contemplando o saber popular como objeto de estudo e, assim, promover um atendimento humanizado e qualificado.

\section{REFERÊNCIAS}

1.Ministério da Saúde (BR). Atenção à saúde do recém-nascido: guia para os profissionais de saúde. $2^{a}$ edição. Volume: 2. Brasília (DF): Ministério da Saúde; 2014.

2. Gaiva MAMG, Fujimori E, Sato APS. Mortalidade neonatal: análise das causas evitáveis. Rev. enferm. UERJ. 2015;23(2):247-53.

3. Andrade LFB, Rodrigues QP, Silva RCV. Boas Práticas na atenção obstétrica e sua interface com a humanização da assistência. Rev. enferm. UERJ. [Internet]. [citado em 05 set. 2019]; 5 (1): 90-96. doi: https://doi.org/10.12957/ reuerj.2017.26442.

4. Ministério da Saúde (Br). Portaria no 1.459, de 24 de junho de 2011. Institui, no âmbito do Sistema Único de Saúde a Rede Cegonha. Brasília (DF): Ministério da Saúde; 2011. 5.Hockenberry MJ, Wilson D. Fundamentos de enfermagem pediátrica-Wong. 9a ed. Rio de Janeiro (RJ): Elsevier; 2014. $1176 \mathrm{p}$.

6.Khan SM, Kim ET, Sing K, Amazou A, Carvajal-Aquise L. Thermal care of newborn: drying and bathing practices in Malawi and Bangladesh. J Glob. Health. 2018; 8(1) 010901.

7.Ministério da Saúde (BR). Portaria n.² 2068, de 21 de outubro de 2016. Institui diretrizes para a organização de atenção integral e humanizada à mulher e ao Recém-nascido no alojamento conjunto. Brasília (DF): Ministério da Saúde; 2016. 


\section{artigo}

Cunha, A.L.L.; Oliveira, S.G.; Bernardo da Silva, M.R.; Chicharo, S.C.R.; Medeiros, C.S.; Christoffel, M.M.; Silva dos Santos, K.C.F.

Práticas culturais do primeiro banho do recém-nascido na maternidade: um estudo etnográfico

\section{REFERÊNCIAS}

8.Sand I, Monticelli M, Ressel LC, Bretas A, Schimmer J. Antropologia da saúde: contribuições teóricas para a interpretação do processo do nascimento. Rev. enferm. UFPE on line. [Internet]. 2014 [citado em 5 set. 2019]; 8(8):2896906. Disponivel em: http: /www.researchgate.net/publication/271764405.

9.Priore MD. História das crianças no Brasil. 7. ed. São Paulo:Contexto; 2016. 504 p.

10.Vargens OMC, Silva CA, Progianti JM. Contribución de enfermeras obstétricas para la consolidación del parto humanizado en maternidades en Río de janeiro -Brasil. Esc. Anna Nery Rev. Enferm. 2017; 21(1):1414-24.

11.Ministério da Saúde (BR). Protocolo clínico e diretrizes terapêuticas para manejo da infecção pelo HIV em crianças e adolescentes. Brasília-DF:2017

12. World Health Organization (WHO). Recomendations on pos natal care of the mother and newborn [Internet]. 2013 [cited 2019 jan 18]; Available from: http://apps.who.int/ iris/bitstream/10665/97603/1/978924241506649.pdf

13.Carvalho VO, Markus JR, Abagge KT, Geraldi S, Campos T. Consenso de Cuidado com a pele do Recém-Nascido [Internet]. São Paulo: SBP; 2015 [cited 2019 jan 18]. Disponivel em: http://www.sbp.com.br.

14.Dyer JA. Newborn skin care. Semin perinatal [Internet]. 2013 [cited 2019 jan 20]; 37(1):3-7. Doi: http://dx.doi. org/10.1053/S0103-201211008.

15.Bozzano AN, Taub L, Overhelman R, Var Cheron L. Newborn care in the home and health facility: formative findings for intervention research in Cambodia. Health Care [Internet]. 2016 [cited 2019 20]; 4(4): 94-99. Doi: http:// dx.doi.org/10.3390/4040094.

16. Ministério da Saúde (BR). Secretaria de Atenção à Saúde. Portaria n. ${ }^{\circ} 371$ de 07 de maio de 2014, institui diretrizes para a organização da atenção integral e humanizada ao Recém-nascido (RN) no Sistema Único de Saúde (SUS). Brasília (DF): 2014.

17. Minayo MCS. O desafio do conhecimento: pesquisa qualitativa em saúde. 14. ed. São Paulo: Hucitec; 2014.

18.Geertz C. Obras e vidas: o antropólogo como autor. 2. ed. Rio de Janeiro: UFRJ; 2005. 134 p.

19. Oliveira RC. O trabalho do antropólogo: olhar, ouvir, escrever. In: 0 trabalho do Antropólogo. 3. ed. São Paulo (SP): Unesp; 2006. 242 p.

20.Minayo MCS. A produção de conhecimentos na interface entre as ciências sociais e humanas e a saúde coletiva. Saude soc. 2013; 22(1):21-31.

21.Pacheco STA, Rodrigues BMRD, Bertolassi BMA. A promoção do cuidado materno ao neonato prematuro: a perspectiva da educação problematizadora em saúde. Rev. enferm. UERJ [Internet]. 2015 [citado em 2019 Jan 20]; 23(1):128-31. Disponivel em: http://www.facenf.uerj.br/ v23n1/v23n1a21.pdf.

22. Dulfe PAM, Aguiar RCB, Alves HW. O cuidado de enfermagem na admissão e permanência do recém-nascido no alojamento conjunto na transferência intra-hospitalar. Rev. Pesqui. Cuid. Fundam. [Internet]. 2015 [citado em 2019 set 5]; 7(2):2287-2297. Disponivel em: https://periodicos.ufpe.br/revistas/revistaenfermagem/article/viewFile/9705/9774.

23.Caldas JP, Millen FC, Camargo JF, Castro PA, Camilo AL, Marbas ST. Effectiveness of a measure program to prevent admission hypothermia in very low-birth weight preterm infants. J. Pediatr. 2018; 94:368-74.

24.Ferreira C S, Gárcia ESGF, Clapis MJ, Carneiro PAP, Moreira D da S, Felipe AOB. O recém-nascido com hipoglicemia e suas respectivas mães: experiência de um alojamento. Rev. Nursing- Edição Portuguesa. 2017.

25.Boiten WA, Berkers T, Absalah S, Van Smeden J, Lavrijsen APM, Boustra JA. Applying a vernix caseosa based formulation accelerates skin barrier repair by modulating lipid biosynthesis. Journal of lipid research. 2018; 59(2):250260.

26.Taieb A. Skin barrier in the neonate pediatric dermatology. Pediatr Dermatol [Internet]. 2018 [cited 2019 jan 18]; 35 (1):55-59. DOI: 10.1111/pde.13482.

27.Association of Women's Health Obstetric and Neonatal Nurses. Neonatal skin care: Evidence-based clinical practice. Guideline. (4th ed.) 2018. 324 p.

28.Agency for Healthcare Research and Quality. Guideline summary NGC-10309. recommendations on postnatal care of the mother and newborn. 2014.

29.Preer G, Pisenga JM, Cook JT, Henri AM, Philipp BL. Delaying the bath and in-hospital breastfeeding rates. Breastfeeding Medicine. 2013; 8:485-490.

30.Andy C, Bena JF, Albert Nm. Initiative to improve exclusive breast feeding by delaying the newborn bath. Journal of obstetric, gynecologic \& neonatal nursing-JOGNN. 2019; 48(2):189-196.

31. Association of Women's Health Obstetric and Neonatal Nurses. Neonatal skin care: Evidence-based clinical practice. Guideline. (3th ed.) 2014.

32. Albuquerque RS, Mariani Neto C, Bersusa AAB, Dias VM, Silva MIM. Newborns temperature submitted to radiant heat and to the top maternal device at birth. Rev. Latino-Am. enferm. 2016; 24: e2741.

33. Oliveira EAR, Rocha SS. 0 cuidado cultural às crianças na dinâmica familiar: reflexões para a enfermagem. R. Interd. 2015; 8(1):227-233.

34. Silva MCS, et.al. Sentimentos vivenciados por puérperas na realização do primeiro banho do RN. O mundo da saúde. 2015; 39(3):279-286. 